\title{
Young and exhausted
}

\author{
Filip Olekšák ${ }^{1}$, Peter Durdik ${ }^{1}$, Ľubica Jakušová ${ }^{1}$, Tomáš Turčan ${ }^{1}$, and Peter Banovcin ${ }^{1}$ \\ ${ }^{1}$ University Hospital Martin
}

June 22, 2020

\begin{abstract}
Chronic Fatigue Syndrome (CFS), also known as myalgic encephalomyelitis (ME), is a complicated disease characterized by extreme fatigue of at least six months duration. It is necessary to rule out the organic cause of CFS in the paediatric patient in order not to overlook the preventable or the treatable condition. In our case report we present a case of a patient with a surprising origin diagnosed by cardiopulmonary exercise testing.
\end{abstract}

\section{Young and exhausted}

Olekšák F, Ďurdík P, Jakušová Ľ, Turčan T, Bánovčin P

Clinic for children and adolescents, University hospital Martin, Slovakia

Jessenius medical faculty in Martin, Commenius University in Bratislava, Slovakia

Chronic Fatigue Syndrome (CFS), also known as myalgic encephalomyelitis (ME), is a complicated disease characterized by extreme fatigue of at least six months duration. It is necessary to rule out the organic cause of CFS in the paediatric patient in order not to overlook the preventable or the treatable condition. In our case report we present a case of a patient with a surprising origin diagnosed by cardiopulmonary exercise testing.

key words: chronic fatigue syndrome, dysfunctional breathing, cardiopulmonary exercise testing

\section{Introduction}

Chronic Fatigue Syndrome/Myalgic Encephalomyelitis (CFS/ME) is a complex, multisystem and often debilitating disorder of unknown etiology. (1) It is a complicated disease characterized by at least six months (in paediatrics 3 months) of extreme fatigue that is not alleviated by rest and a group of other symptoms that are constant for a period of time. (1)

Post-exertional malaise (PEM) and delayed recovery are core symptoms and the most useful when making a diagnosis. (3) PEM involves a constellation of substantially disabling signs and symptoms that occur in response to physical, mental, emotional, and spiritual over-exertion. (2) In many people with chronic fatigue syndrome, the disorder begins suddenly, often after a flu-like infection or after an episode of physical or mental trauma. The diagnosis of CFS/ME relies on the typical clinical presentation and the exclusion of other causes of fatigue. A diagnosis can be made by taking a thorough history, examining the patient and using blood tests to screen for other causes of fatigue. (3) Up until now there was no test to confirm the diagnosis of CFS/ME. The two-day cardiopulmonary exercise testing (CPET) is becoming a new diagnostic method that can be used in case of suspicion of CFS/ME in attempt to evaluate presence of PEM. (5) This test, used in centres for patients with CFS/ME, has not yet published any data in paediatrics to support its use. CPET, on the other hand, provides a wealth of data on the dynamic function and coordination 
of the heart, lungs and muscles, as well as on the efficiency of gas exchange between mitochondria and the surrounding air, even in patients complaining about PEM.

\section{Case report}

In the case report, we present a case of a 13-year-old patient with chronic debilitating fatigue who meets the criteria for CFS/ME. The patient was examined in detail by a paediatrician (anamnestic unclear cause, resting tachycardia in the physical examination, laboratory tests within normal limits), endocrinologist (normal hormonal profile for a given age, Tanner stage 3), infectologist (serology for typical viruses negative), psychologist (normal cognitive functions). For a history of resting tachycardia, the patient was examined by a cardiologist where no cardiogenic cause of fatigue was demonstrated, sinus tachycardia was present, and the patient was recommended head-up-tilt test, which showed the presence of postural orthostatic tachycardia. Due to the idiopathic nature of the difficulties and the excluded secondary cause, a two-day protocol examination by cardiopulmonary exercise testing was indicated. Written consent was taken and documented. Before the examination, resting spirometry (physiological findings) was performed, resting ECG (sinus tachycardia) and a shortened Schellong test with a tachycardic response to orthostasis immediately after standing up and after 3 minutes of standing. Subsequently, standard CPET using a treadmill (Itam, Poland) with individualized protocol with progressive increase in workload until exhaustion and breath-by-breath analysis of exhaled gases (Geratherm, Germany) was performed. On the first day, basal values were determined, the patient subjectively tolerated the examination well, the exercise ended prematurely due to subjective fatigue and a feeling of lack of air. On the second day, under the same conditions, CPET was repeated, during which the patient also terminated the exercise prematurely, but a half minute later than on the first day. Using this methodology, the patient did not meet the diagnostic criteria for PEM and subsequently CFS/ME (decrease in monitored values on the second day of examination) (table 1). On both days, during exercise, a bizarre pattern of respiration with malposition of the respiratory act to the large airways was observed by observing the flow-volume loop. An analysis of the respiratory pattern identified an erratic respiratory pattern (figure 1a) with low resting ETCO2 (table 1) and tachypnoea at maximal workload with dominant ventilation of dead space (figure 1b, 1c). The consequence of this pattern of respiration is a chronic state of hypocapnia and respiratory alkalosis, which is metabolically compensated in the patient. Fatigue and increased heart rate are expected clinical manifestations. Respiratory rehabilitation was recommended to the patient in order to fixate the correct breathing patterns (diaphragmatic breathing) and psychological guidance. The patient was subsequently retested 3 months after the start of physiotherapy and psychotherapy at the request of the parents. Retesting showed significant improvements in the monitored parameters (table 1) as well as in the clinical condition of the patient. The patient's overall fitness increased, an adequate resting respiratory pattern was present (figure 1d), ventilatory efficiency was adjusted (VE/VCO2 in table 1, figure 1f), and the patient reported a subjective increase in energy. Prior to the examination, we performed a Schellong test on the patient, in which there were no signs of postural orthostatic tachycardia.

\section{Discussion}

Dysfunctional breathing (DB) is a condition of the airways characterized by an irregular breathing pattern and changes in the airways that cannot be attributed to a specific diagnosis and that causes respiratory and non-respiratory problems. (4) It is not a disease process, but rather changes in respiratory patterns that disrupt normal respiratory processes. However, DB can coexist with diseases such as bronchial asthma or heart disease. The main symptom is shortness of breath or air hunger, associated with non-respiratory symptoms such as dizziness, palpitations, cervical spine pain or fatigue. (6) It also plays a role in chronic fatigue, neck and back pain, fibromyalgia, and some aspects of anxiety and depression. (6)

The most common type of $\mathrm{DB}$ is hyperventilation syndrome, which is defined as respiration exceeding metabolic requirements, reducing blood carbon dioxide concentrations below normal values. (4) This changes the $\mathrm{pH}$ of the blood, increases the alkalinity and thus triggers a number of adaptive changes that cause symptoms. These conditions are non-somatic in nature and their treatment consists of respiratory rehabilitation by various techniques (diaphragmatic breathing, Feldenkrais method, Buteyko method, Pilates) and psychotherapy in order to control impulsive changes in the respiratory pattern in various situations. (6) 


\section{Conclusion}

CPET confirmed the presence of DB in the patient based on the low resting value of ETCO2, the presence of a chaotic pattern of respiration during resting and exercise with the presence of tachypnoea (with very low ventilatory efficiency) in maximal exertion. Diagnosis of DB using CPET is one of the methods of DB diagnostics. Proper respiratory rehabilitation and psychological guidance resulted in the patient fixing the respiratory pattern and subsequently eliminating the primary cause of the examination - chronic fatigue. Patients with CFS/ME are a common paediatric problem. The current possibilities of diagnostics are enriched by the possibility of performing CPET, which can be a benefit in differential diagnostics as well as in confirming the diagnosis.

\section{Conflict of interest}

No conflict of interest to declare.

\section{Sources}

1. Clayton EW. Beyond Myalgic Encephalomyelitis/Chronic Fatigue Syndrome. Jama. 2015;313(11):1101. doi:10.1001/jama.2015.1346 2. Davenport TE, Lehnen M, Stevens SR, Vanness JM, Stevens J, Snell CR. Chronotropic Intolerance: An Overlooked Determinant of Symptoms and Activity Limitation in Myalgic Encephalomyelitis/Chronic Fatigue Syndrome? Frontiers in Pediatrics. 2019;7. doi:10.3389/fped.2019.00082 3. Baker R, Shaw EJ. Diagnosis and management of chronic fatigue syndrome or myalgic encephalomyelitis (or encephalopathy): summary of NICE guidance. Bmj. 2007;335(7617):446-448. doi:10.1136/bmj.39302.509005.ae 4. Boulding R, Stacey R, Niven R, Fowler SJ. Dysfunctional breathing: a review of the literature and proposal for classification. European Respiratory Review. 2016;25(141):287-294. doi:10.1183/16000617.0088-2015 5. Stevens S, Snell C, Stevens J, Keller B, Vanness JM. Cardiopulmonary Exercise Test Methodology for Assessing Exertion Intolerance in Myalgic Encephalomyelitis/Chronic Fatigue Syndrome. Frontiers in Pediatrics. 2018;6. doi:10.3389/fped.2018.00242 6. Chaitow L, Bradley D, Gilbert C, Bartley J, Peters D. Recognizing and treating breathing disorders: a multidisciplinary approach. Edinburgh, Scotland: Churchill Livingstone; 2014.

\section{Author for correspondence}

Peter Ďurdík, MD., PhD., Clinic for children and adolescents, University hospital Martin, Slovakia

Jessenius medical faculty in Martin, Commenius University in Bratislava, Kollárova 2, 03659 Martin, Slovakia e-mail: peter.durdik@unm.sk

\section{Hosted file}

Table 1.docx available at https://authorea.com/users/335130/articles/461016-young-andexhausted 

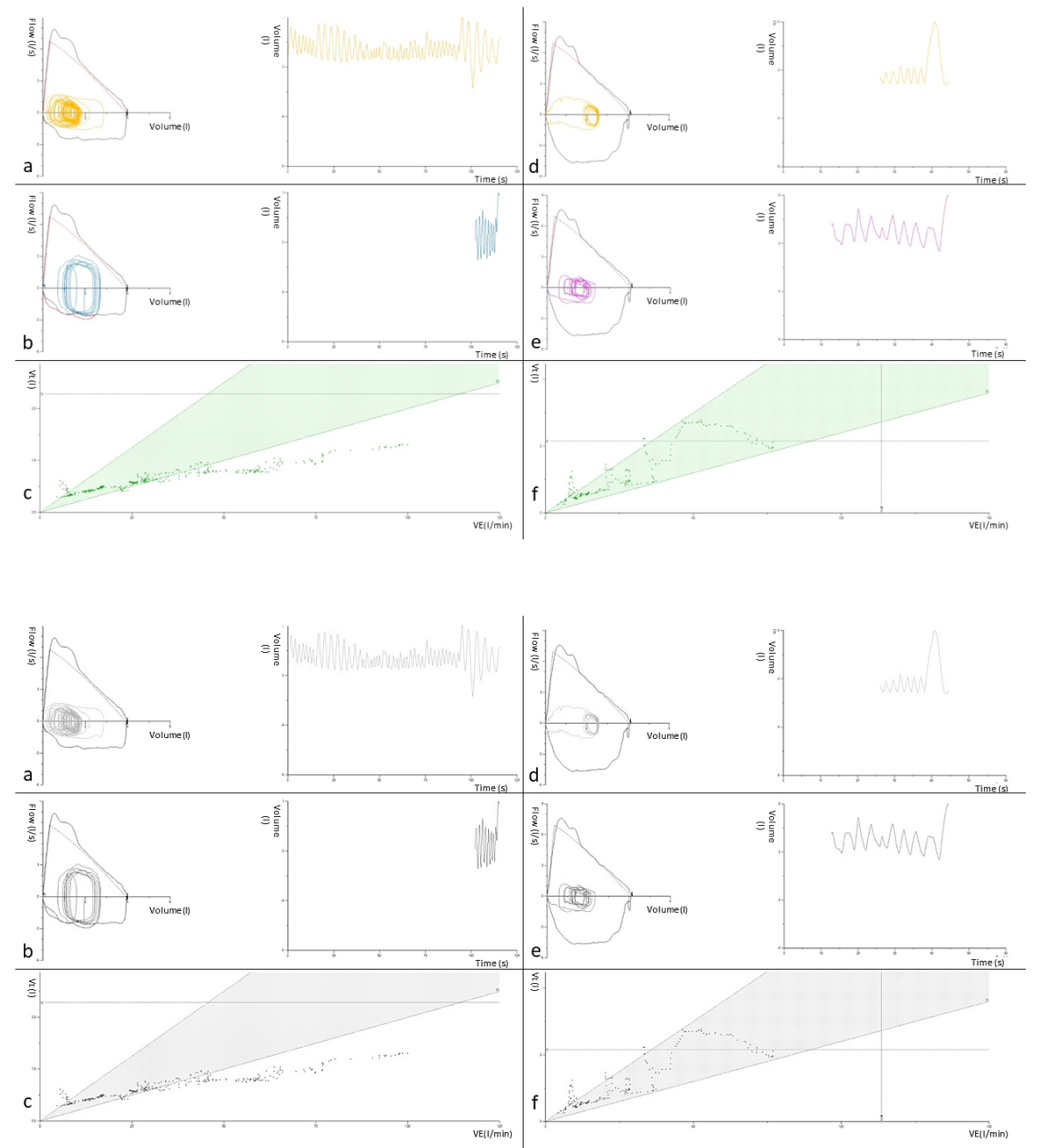